\title{
Effect of CYP2C19 and CYP3A4 gene polymorphisms on the efficacy of bortezomib-based regimens in patients with multiple myeloma
}

\author{
WEIWEI ZHOU ${ }^{1}$, GUANGYU AN ${ }^{1}$, YUAN JIAN ${ }^{2}$, HUAN GUO ${ }^{2}$ and WENMING CHEN ${ }^{2}$ \\ Departments of ${ }^{1}$ Oncology and ${ }^{2}$ Hematology, Beijing Chao-Yang Hospital, \\ Capital Medical University, Beijing 100020, P.R. China
}

Received July 8, 2014; Accepted March 27, 2015

DOI: $10.3892 / \mathrm{ol} .2015 .3294$

\begin{abstract}
Bortezomib is used to treat patients with multiple myeloma. It is primarily metabolized by the enzyme cytochrome P450 (CYP). Variations in the capacity of bortezomib metabolism affect the treatment outcomes and the side-effects experienced by patients. In the present study, polymorphisms in the CYP3A4 and CYP2C19 genes were analyzed by polymerase chain reaction in 56 newly-diagnosed patients with multiple myeloma. The polymorphisms analyzed included the c.681G >A, c.636G $>\mathrm{A}$ and c.-806C $>\mathrm{T}$ polymorphisms of CYP2C19. The CYP3A4 gene was sequenced after amplification and was classified into normal and mutant types. Associations between the metabolizer genotypes of CYP3A4 and CYP2C19, the therapeutic efficacy of bortezomib-based regimens, and the occurrence of peripheral neuropathy were studied. The results identified no significant differences in gender, serum $\beta 2$ microglobulin, creatinine, blood albumin, isotypes, and the Durie-Salmon and International Staging System stages between the CYP2C19 poor + intermediate metabolizer types and the extensive + ultrarapid metabolizer types. In addition, it was revealed that the CYP2C19 and CYP3A4 phenotypes did not affect the efficacy of bortezomib-based regimens, nor were they correlated with peripheral neuropathy. Additional large-scale studies are required in order to evaluate the role of CYP enzymes in bortezomib treatments for patients with multiple myeloma.
\end{abstract}

\section{Introduction}

Bortezomib is a therapeutic proteasome inhibitor that belongs to a novel class of active agents used for the treatment of

Correspondence to: Professor Wenming Chen, Department of Hematology, Beijing Chao-Yang Hospital, Capital Medical University, 8 Gongren Tiyuchang Nanlu, Beijing 100020, P.R. China E-mail: 13910107759@163.com

Key words: bortezomib, CYP2C19, CYP3A4, polymorphism, peripheral neuropathy newly-diagnosed and relapsed/refractory multiple myeloma (1). The response to bortezomib and the side-effects experienced varies between patients, which suggests that individual differences have an effect. The principal biotransformation pathway of bortezomib is the oxidative deboronation of cytochrome P450 (CYP) (2). Therefore, variations in CYP genes may alter the activities of the enzymes, result in altered pharmacokinetics of bortezomib and affect the outcome of treatment (3). Studies concerning genotype-based drug utilization may present a promising future for individualized therapy. CYPs are encoded by 57 genes and, in total, comprise 50 families and subfamilies of enzymes (4). CYP3A4, CYP2C19, CYP2D6, CYP1A2 and CYP2C9 are major isoforms that contribute to the metabolism of bortezomib. A previous study identified the percentage of each isoform to be 69.8, 33.5, 23.4, 15.8 and $13.6 \%$, respectively (5). CYP3A4 and CYP2C19 are considered to be the principal metabolic enzymes for the inactivation of bortezomib (5).

Polymorphisms in the CYP genes have been extensively studied. Patients can be categorized into four types as follows: i) Poor metabolizer (PM); ii) intermediate metabolizer (IM); iii) extensive metabolizer (EM); and iv) ultrarapid metabolizer (UM) (4). Previous studies have reported an association between CYP genes and the drugs used to treat multiple myeloma. Specifically, the metabolisms of certain drugs (such as cyclophosphamide and thalidomide) have been reported to be affected by CYP polymorphisms. Timm et al (6) revealed that the pharmacokinetics of cyclophosphamide are significantly affected by CYP2C19*2 genotype. An additional study found that myeloma patients with the CYP2C19 PM genotype responded poorly to treatment with thalidomide (7). With respect to bortezomib, a study consisting of 348 Caucasian patients identified no association between the functional CYP2C19 and CYP2D6 alleles and the treatment outcome in patients with multiple myeloma that were treated with bortezomib, thalidomide or cyclophosphamide (8). Limited data exists concerning the association between CYPs and bortezomib in Asian populations.

In the present study, polymorphisms in the CYP3A4 and CYP2C19 genes were analyzed by polymerase chain reaction (PCR) in 56 newly-diagnosed patients with multiple myeloma. The aim of the current study was to elucidate the association 
between the metabolizer genotypes and the therapeutic efficacy of bortezomib-based regimens, as well as the occurrence of peripheral neuropathy (PN), which is the main side-effect of bortezomib treatment.

\section{Materials and methods}

Patients. In total, 56 patients with newly-diagnosed multiple myeloma were recruited between May 2013 and December 2013 at the Beijing Chao-Yang Hospital affiliated to the Capital Medical University (Beijing, China). All patients were administered with bortezomib-based regimens, and peripheral blood samples were drawn prior to the start of the combined therapy. In addition, information concerning the clinical history, diagnosis, treatment and outcomes was obtained for each of the patients. The serum $\beta 2$ microglobulin, creatinine and blood albumin levels at the time of diagnosis were also collected. Staging was assigned according to the Durie and Salmon (DS) and the International Staging System (ISS) criteria (9).

Patients were administered with one of the following bortezomib-based regimens: i) PAD, consisting of bortezomib, epirubicin and dexamethasone; ii) PCD, consisting of bortezomib, cyclophosphamide and dexamethasone; or iii) PTD, consisting of bortezomib, thalidomide and dexamethasone. Bortezomib was administered at a dose of $1.3 \mathrm{mg} / \mathrm{m}^{2}$ on days $1,4,8$ and 11 , epirubicin at $20 \mathrm{mg}$ on days 1-4, dexamethasone at $20 \mathrm{mg}$ on days 1-2, 4-5, 8-9 and 11-12, and thalidomide at $100 \mathrm{mg} /$ day. Patient responses were evaluated every two cycles. The responses, which were based on the International Myeloma Working Group uniform criteria (10), were categorized into five groups. A complete response (CR) was defined as a negative immunofixation on the serum and urine, the disappearance of any soft-tissue plasmacytomas, and $\leq 5 \%$ plasma cells in the bone marrow. A very good partial response (VGPR) and a partial response (PR) were defined as a reduction in the level of serum M-protein of $>90$ and $>50 \%$, respectively. Progressive disease (PD) was defined as either a $>25 \%$ increase in serum M-protein, urine M-protein or bone marrow plasma cells, an increase in the size and number of bone lesions or tissue plasmacytomas, or the development of hypercalcemia that could be solely attributed to the plasma cell proliferative disorder. Stable disease (SD) was defined as not meeting the criteria for CR, VGPR, PR or PD.

The four phenotypes (PM, IM, EM and UM) of metabolizers were determined by the numbers of functional alleles, which have normal or increased activity. The genotype of a PM was a homozygous variant with the absence of a functional protein. The genotype of an IM was a heterozygous variant with one functional allele. The genotype of an EM was a homozygous wild-type with a normal enzymatic capacity, and the genotype of a UM was a duplication of functional genes (4).

The incidence of $\mathrm{PN}$, which is the main side effect of treatment with thalidomide or bortezomib, was noted in the present study. The degree of PN was established according to the following criteria: i) Level 1, no dose reduction at treatment; ii) level 2 , dose reduction due to neurological adverse reactions; and iii) level 3 , cessation of treatment due to neurological adverse reactions (8).

The present study was approved by the Ethics Committee of Beijing Chao-Yang Hospital, Capital Medical University.
Written informed consent was obtained from the family of each patient.

Methods. DNA was extracted from peripheral blood samples, amplified using a PCR kit (Takara Biotechnology Co., Ltd., Dalian, China) and then sequenced by Sanger methods. The total reaction volume used in PCR was $50 \mu \mathrm{l}$, which included: 10X amplification buffer solution $(5 \mu \mathrm{l})$, dNTP $(4 \mu \mathrm{l})$, forward primer $(2 \mu 1)$, reverse primer $(2 \mu 1)$, template DNA $(1 \mu 1)$, Taq DNA $(0.5 \mu \mathrm{l})$, and super-purified water. The PCR reaction conditions for the two primer sets were as follows: $98^{\circ} \mathrm{C}$ for $2 \mathrm{~min}$, followed by 25 cycles consisting of $96^{\circ} \mathrm{C}$ for $10 \mathrm{sec}, 50^{\circ} \mathrm{C}$ for $5 \mathrm{sec}$, and $60^{\circ} \mathrm{C}$ for $4 \mathrm{~min}$. The results were compared with the database of CYPs (11), and the haplotypes of CYP2C19 and CYP3A4 were obtained. The phenotypes of the metabolizers were determined by the number of functional alleles.

Statistical analysis. Statistical analysis was performed using SPSS 17.0 software (SPSS, Inc., Chicago, IL, USA). The measurement data was calculated using an independent samples $t$-test. The categorical data was calculated using the $\chi^{2}$ test. $\mathrm{P}<0.05$ was considered to indicate a statistically significant difference.

\section{Results}

Overall, 56 patients with newly-diagnosed multiple myeloma were included in the present study. The patients consisted of 24 males and 32 females, with a median age of 56.5 years (range, 33-79 years). The PAD, PCD and PTD regimens were administered to $34(60.7 \%), 16(47.1 \%)$ and $6(17.6 \%)$ patients, respectively. Of these 56 patients, 22 (39.3\%) achieved CR, $10(17.9 \%)$ demonstrated PR, $8(14.3 \%)$ demonstrated SD and 8 (14.3\%) experienced PD.

The CYP2C19 and CYP3A4 genes were analyzed in 56 peripheral blood samples. The definitions of PM, IM, EM or UM were different according to the CYPs. For CYP2C19, the genotype ${ }^{*} 1 /{ }^{*} 1$ is classified as EM, ${ }^{*} 1 /{ }^{*} 3$ or ${ }^{*} 1 /{ }^{*} 2$ as IM, ${ }^{*} 2 /{ }^{*} 2$ or *2/3 as PM, and *1/*17 as UM. However, no definite classification of CYP3A4 genotype was reported. The patients in the present study were divided into two groups (normal and mutated), according to the CYP3A4 genotype. For CYP2C19, the alleles present were ${ }^{*} 1 /{ }^{*} 1$ in 21 patients (EMs), ${ }^{*} 1 /{ }^{*} 3$ or ${ }^{*} 1 /{ }^{*} 2$ in 26 patients (IMs), ${ }^{*} 2 /{ }^{*} 2$ or ${ }^{*} 2 /{ }^{*} 3$ in 8 patients (PMs) and ${ }^{*} 1 /{ }^{*} 17$ in 1 patient (UM). The frequencies of the alleles, ${ }^{*} 1,{ }^{*} 2$, *3 and *17, were $61.6,33.0,3.65$ and $1.8 \%$, respectively.

Association between CYP2C19 phenotypes and patient characteristics and treatment outcome. The associations between the phenotypes of CYP2C19 and the patient characteristics are summarized in Table I. There were no significant differences identified for gender, $\beta 2$ microglobulin, creatinine, albumin, isotype, DS and ISS stage between PM + IM and EM + UM. The response rate $(\mathrm{CR}+\mathrm{VGPR}+\mathrm{PR})$ for $\mathrm{PM}+\mathrm{IM}$ was higher than that of EM + UM (76.5 vs. 63.6\%). However, this difference was not statistically significant $(\mathrm{P}=0.299$; Table II).

Association between CYP3A4 genotypes and treatment outcome. The results of the CYP3A4 gene sequencing revealed that the genotypes of 50 patients were normal, but that 6 patients 
Table I. Association between the phenotypes of CYP2C19 and the characteristics of patients with multiple myeloma.

\begin{tabular}{|c|c|c|c|}
\hline CYP2C19 & $\mathrm{PM}+\mathrm{IM}$ & $\mathrm{EM}+\mathrm{UM}$ & P-value ${ }^{a}$ \\
\hline Total, n (\%) & $34(100.0)$ & $22(100.0)$ & \\
\hline Age, years (range) & $56(33-79)$ & $58(44-77)$ & \\
\hline \multicolumn{4}{|l|}{ Gender, n (\%) } \\
\hline Male & $14(41.2)$ & $10(45.5)$ & \multirow[t]{2}{*}{$0.752^{\mathrm{b}}$} \\
\hline Female & $20(58.8)$ & $12(54.5)$ & \\
\hline$\beta 2$ microglobulin, $\mathrm{mg} / \mathrm{l}$ (range) & $8.92(6.12-11.72)$ & $11.15(5.78-16.52)$ & $0.786^{\mathrm{c}}$ \\
\hline Albumin, g/l (range) & $33.4(31.2-35.6)$ & $31.3(28.1-34.5)$ & $0.274^{\mathrm{c}}$ \\
\hline \multicolumn{4}{|l|}{ Creatinine, n (\%) } \\
\hline$\leq 177.6 \mu \mathrm{mol} / 1$ & $28(82.3)$ & $18(81.8)$ & \multirow[t]{2}{*}{$1.000^{\mathrm{b}}$} \\
\hline$>177.6 \mu \mathrm{mol} / \mathrm{l}$ & $6(17.7)$ & $4(18.2)$ & \\
\hline \multicolumn{4}{|l|}{ Isotype, n (\%) } \\
\hline $\operatorname{IgG}$ & $15(44.1)$ & $6(27.3)$ & \multirow[t]{5}{*}{$0.299^{\mathrm{b}}$} \\
\hline $\operatorname{IgA}$ & $5(14.7)$ & $4(18.1)$ & \\
\hline $\operatorname{IgD}$ & $3(8.9)$ & $6(27.3)$ & \\
\hline Light chains $(\kappa+\lambda)$ & $9(26.5)$ & $6(27.3)$ & \\
\hline Non-secretory & $2(5.9)$ & $0(0.0)$ & \\
\hline \multicolumn{4}{|l|}{ DS stage, n (\%) } \\
\hline $\mathrm{I}$ & $2(5.9)$ & $1(4.5)$ & \multirow[t]{3}{*}{$1.000^{\mathrm{b}}$} \\
\hline II & $2(5.9)$ & $1(4.5)$ & \\
\hline III & $30(88.2)$ & $20(91.0)$ & \\
\hline \multicolumn{4}{|l|}{ ISS stage, n (\%) } \\
\hline I & $10(29.4)$ & $4(18.2)$ & \multirow[t]{3}{*}{$0.533^{\mathrm{b}}$} \\
\hline II & $10(29.4)$ & $6(27.3)$ & \\
\hline III & $14(41.2)$ & $12(54.5)$ & \\
\hline
\end{tabular}

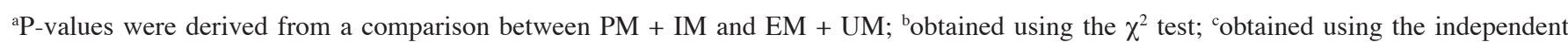
samples t-test. CYP2C19, cytochrome P450 2C19; PM, poor metabolizer; IM, intermediate metabolizer; EM, extensive metabolizer; UM, ultrarapid metabolizer; Ig, immunoglobulin; DS, Durie and Salmon; ISS, International Staging System.

Table II. Association between the phenotypes of CYP2C19 and the outcome of treatment.

\begin{tabular}{lrrr}
\hline & \multicolumn{2}{c}{ CYP2C19 } & \\
\cline { 2 - 3 } Treatment outcome & PM + IM & EM + UM & P-value \\
\hline CR, VGPR or PR, n (\%) & $26(76.5)$ & $14(63.6)$ & 0.299 \\
SD or PD, n (\%) & $8(23.5)$ & $8(36.4)$ &
\end{tabular}

P-value was derived from a comparison between PM + IM and $\mathrm{EM}+\mathrm{UM}$, and was obtained using the $\chi^{2}$ test. CYP2C19, cytochrome P450 2C19; PM, poor metabolizer; IM, intermediate metabolizer; $\mathrm{EM}$, extensive metabolizer; UM, ultrarapid metabolizer; CR, complete response; VGPR, very good partial response; PR, partial response; $\mathrm{SD}$, stable disease; $\mathrm{PD}$, progressive disease.

exhibited genotype mutations. CYP3A4 gene mutations were found in the introns of three samples and in the mRNA of one sample. In addition, a mutation was detected in the exon of one sample; however, it did not result in the alteration of an amino acid. The CYP3A4 genotype was CYP3A4*1*18 in one
Table III. Association between the phenotypes of CYP3A4 and the outcome of treatment.

\begin{tabular}{llll}
\hline & \multicolumn{2}{c}{ CYP3A4 } & \\
\cline { 2 - 3 } Treatment outcome & Normal & Mutant & P-value \\
\hline CR, VGPR or PR, n (\%) & $37(74.0)$ & $4(66.7)$ & 0.654 \\
SD or PD, n $(\%)$ & $13(26.0)$ & $2(33.3)$ & \\
& & &
\end{tabular}

P-value was derived from a comparison between normal and mutant type CYP3A4, and was obtained using the $\chi^{2}$ test. CYP3A4, cytochrome P450 3A4; CR, complete response; VGPR, very good partial response; $\mathrm{PR}$, partial response; $\mathrm{SD}$, stable disease; $\mathrm{PD}$, progressive disease.

sample. The response rates of patients with normal and mutant CYP3A4 genotypes were $74.0 \%$ and $66.7 \%$, respectively. This difference was not statistically significant $(\mathrm{P}=0.654$; Table III).

Association between CYP2C19 and CYP3A4 phenotypes and treatment outcome. Combinations of the CYP2C19 and 
Table IV. Association between the phenotypes of CYPs and the outcome of treatment.

\begin{tabular}{lcc}
\hline Treatment outcome & $\begin{array}{c}\text { CYP2C19 EM + UM } \\
\text { with mutant CYP3A4 }\end{array}$ & $\begin{array}{c}\text { CYP2C19 PM + IM } \\
\text { with normal CYP3A4 }\end{array}$ \\
\hline CR, VGPR or PR, n (\%) & $0(0.0)$ & $23(76.7)$ \\
SD or PD, n $(\%)$ & $2(100.0)$ & $7(23.3)$ \\
\hline
\end{tabular}

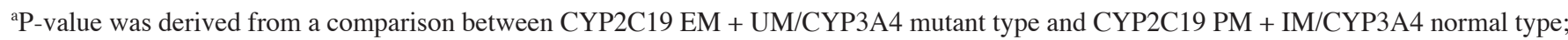
bobtained using the $\chi^{2}$ test. CYP, cytochrome P450; PM, poor metabolizer; IM, intermediate metabolizer; EM, extensive metabolizer; UM, ultrarapid metabolizer; CR, complete response; VGPR, very good partial response; PR, partial response; SD, stable disease; PD, progressive disease.

Table V. Association between phenotypes of CYPs and the outcome of treatment.

\begin{tabular}{lcc}
\hline Treatment outcome & $\begin{array}{c}\text { CYP2C19 EM + UM } \\
\text { with normal CYP3A4 }\end{array}$ & $\begin{array}{c}\text { CYP2C19 PM + IM } \\
\text { with mutant CYP3A4 }\end{array}$ \\
\hline CR, VGPR or PR, n (\%) & $14(70.0)$ & $4(100.0)$ \\
SD or PD, n $(\%)$ & $6(30.0)$ & $0(0.0)$
\end{tabular}

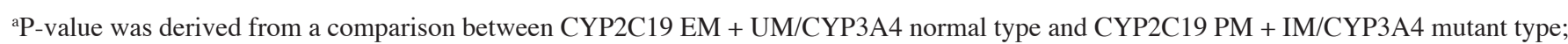
bobtained using the $\chi^{2}$ test. CYP, cytochrome P450; PM, poor metabolizer; IM, intermediate metabolizer; EM, extensive metabolizer; UM, ultrarapid metabolizer; CR, complete response; VGPR, very good partial response; PR, partial response; $\mathrm{SD}$, stable disease; PD, progressive disease.

Table VI. Association between phenotypes of CYPs and PN.

\begin{tabular}{lccccc}
\hline & \multicolumn{2}{c}{ CYP2C19 } & & \multicolumn{2}{c}{ CYP3A4 } \\
\cline { 2 - 3 } PN level & PM + IM, n (\%) & EM + UM, n (\%) & P-value & Normal type, n (\%) & Mutant type, n (\%) $^{\text {P-value }}$ \\
\hline $0-1$ & $30(88.2)$ & $19(86.4)$ & $0.626^{c}$ & $44(88.0)$ & $5(83.3)$ \\
2 & $4(11.8)$ & $2(9.1)$ & & $5(10.0)$ & $0.569^{c}$ \\
3 & $0(0.0)$ & $1(4.5)$ & & $1(2.0)$ & $0(0.0)$ \\
\hline
\end{tabular}

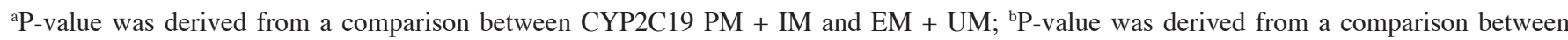
CYP3A4 normal type and mutant type; 'obtained using the $\chi^{2}$ test. CYP, cytochrome P450; PN, peripheral neuropathy; PM, poor metabolizer; IM, intermediate metabolizer; EM, extensive metabolizer; UM, ultrarapid metabolizer.

CYP3A4 phenotypes were analyzed with regard to the outcome of treatment as follows: i) EM + UM for CYP2C19 + mutant type for CYP3A4 compared with PM + IM for CYP2C19 and normal type for CYP3A4; and ii) EM + UM for CYP2C19 + normal type for CYP3A4 compared with $\mathrm{PM}+\mathrm{IM}$ for CYP2C19 + mutant type for CYP3A4. There were no significant differences in the treatment outcomes of patients carrying the various combinations of CYP2C19 and CYP3A4 (Tables IV and V).

Association between phenotypes of CYPs and the occurrence of $P N$. In the present study, one patient ceased bortezomib-based therapy due to serious $\mathrm{PN}$, and six patients with level 2 PN were prescribed a decreased dose of bortezomib. In total, 49 patients experienced either level $1 \mathrm{PN}$ or no PN at all. The association between the phenotypes of the CYPs and the occurrence of PN is shown in Table VI.
No significant differences were found between PM + IM and $\mathrm{EM}+\mathrm{UM}$ for CYP2C19, and normal and mutant types for CYP3A4 ( $\mathrm{P}=0.626$ and $\mathrm{P}=0.569$, respectively).

\section{Discussion}

Polymorphisms in CYP genes may affect the activity of the transcribed enzymes and therefore result in alterations in the metabolism of bortezomib. The present study investigated genetic polymorphisms in the CYP3A4 and CYP2C19 genes, and analyzed the associations between metabolizer genotypes and the outcome of treatment with bortezomib-based regimens.

A trend towards a better outcome in response to bortezomib-based treatment was observed in carriers of CYP2C19 PM + IM. This may reflect a slower metabolism of the drug in these patients. There was no significant effect of CYP2C19 genotype on the response to treatment. 
CYP3A4 is considered to be a major contributor to the metabolism of bortezomib (5). In the present study, six patients were found to carry mutations in the CYP3A4 gene. Non-specific mutations were observed in five patients, whilst the " $1 /{ }^{*} 18$ genotype was present in one patient. CYP3A4" 1 was considered to be the wild type genotype. In a previous study, *18 was only reported in Asian populations, and had a frequency of $2 \%$ (12). The frequency of the CYP $3 A 4 * 18$ allele in the present study was $0.9 \%$. CYP3A4*18 has been identified to be a two-faced mutation with respect to metabolic activity; it acted as a rapid metabolizer of sex steroids, but was a poor metabolizer of other drugs, including midazolam (13). It has been revealed that variants in the coding regions of CYP3A4 occur at allele frequencies of $<5 \%$ and appear in a heterozygous manner with the wild-type allele. Therefore, alterations in the enzymatic activity of CYP3A4 were limited (14). The present study analyzed the association between the phenotypes of CYP3A4 and the outcomes of treatment. However, no significant differences were observed. A combination analysis of polymorphisms in CYP3A4 and CYP2C19 was performed, but no differences were found in treatment outcomes in relation to genotype combinations.

Therefore, it is hypothesized that polymorphisms of CYP may contribute to, but are not the unique factor in, the inter-individual differences in the outcome of treatment. Several factors, including the biological aspects of tumors, tumor burden at diagnosis, immune response and variations in the genes involved in DNA repair or inflammation may be included (8,15-17).

$\mathrm{PN}$ is the main dose-limiting toxicity associated with bortezomib therapy (18). The results of the present study indicated that no differences were found between the occurrence of PN in patients with the CYP2C19 genotype and those with the CYP3A4 genotypes. This result was consistent with that of a previous study by Vangsted et al (8). In addition, another study demonstrated no association with the presence of PN induced by bortezomib, but did establish a significant association between single nucleotide polymorphisms of the TCF4 gene and the time to onset of PN (4).

In conclusion, the present study found no association between CYP2C19 and CYP3A4 genotypes and the outcome of treatment with bortezomib-based regimens in patients with multiple myeloma. Additional studies that include large numbers of patients in controlled clinical trials and meta-analyses are required in order to elucidate the association between CYP enzymes and treatment with bortezomib for patients with multiple myeloma.

\section{References}

1. Chen D, Frezza M, Schmitt S, Kanwar J and Dou QP: Bortezomib as the first proteasome inhibitor anticancer drug: current status and future perspectives. Curr Cancer Drug Targets 11: 239-253, 2011.
2. Kane RC, Bross PF, Farrell AT and Pazdur R: Velcade: U.S. FDA approval for the treatment of multiple myeloma progressing on prior therapy. Oncologist 8: 508-513, 2003.

3. Iyer L and Ratain MJ: Pharmacogenetics and cancer chemotherapy. Eur J Cancer 34: 1493-1499, 1998.

4. Vangsted A, Klausen TW and Vogel U: Genetic variations in multiple myeloma II: association with effect of treatment. Eur J Haemotol 88: 93-117, 2012.

5. Uttamsingh V, Lu C, Miwa G and Gan LS: Relative contributions of the five major human cytochromes P450, $1 \mathrm{~A} 2$, $2 \mathrm{C} 9,2 \mathrm{C} 19,2 \mathrm{D} 6$ and $3 \mathrm{~A} 4$, to the hepaticmetabolism of the proteasome inhibitor bortezomib. Drug Metab Dispos 33: 1723-1728, 2005.

6. Timm R, Kaiser R, Lötsch J, et al: Association of cyclophosphamide pharmacokinetics to polymorphic cytochrome P450 2C19. Pharmacogenomics J 5: 365-373, 2005.

7. Li Y, Hou J, Jiang H, et al: Polymorphisms of CYP2C19 gene are associated with the efficacy of thalidomide based regimens in multiple myeloma. Haematologica 92: 1246-1249, 2007.

8. Vangsted AJ, Søeby K, Klausen TW, et al: No influence of the polymorphisms CYP2C19 and CYP2D6 on the efficacy of cyclophosphamide, thalidomide and bortezomib in patients with multiple myeloma. BMC Cancer 10: 404, 2010.

9. Anderson KC, Bensinger W, Alsina M, et al (eds): Multiple myeloma. In: NCCN Clinical Practice Guidelines in Oncology (NCCN Guidelines ${ }^{\circledR}$ ). Version 1, 2013. http://www.nccn. org/professionals/physician_gls/f_guidelines.asp. Accessed March 12, 2014.

10. Durie BG, Harousseau JL, Miguel JS, et al; International Myeloma Working Group: International uniform response criteria for multiple myeloma. Leukemia 20: 1467-1473, 2006.

11. The Human Cytochrome P450 (CYP) Allele Nomenclature Database. http://www.cypalleles.ki.se/. Accessed 20 Mar, 2014.

12. Dai D, Tang J, Rose R, Hodgson E, Bienstock RJ, Mohrenweiser HW and Goldstein JA: Identification of variants of CYP3A4 and characterization of their abilities to metabolize testosterone and chlorpyrifos. J Pharmacol Exp Ther 299: 825-831, 2001.

13. Kang YS, Park SY, Yim CH, et al: The CYP3A4*18 genotype in the cytochrome P450 3A4 gene, a rapid metabolizer of sex steroids, is associated with low bone mineral density. Clin Pharmacol Ther 85: 312-318, 2009.

14. Lamba JK, Lin YS, Schuetz EG and Thummel KE: Genetic contribution to variable human CYP3A-mediated metabolism. Adv Drug Deliv Rev 54: 1271-1294, 2002.

15. Vangsted AJ, Klausen TW, Ruminski W, et al: The polymorphism IL-1beta T-31C is associated with a longer overall survival in patients with multiple myeloma undergoing auto-SCT. Bone Marrow Transplant 43: 539-545, 2009.

16. Vangsted A, Gimsing P, Klausen TW, et al: Polymorphisms in the genes ERCC2, XRCC3 and CD3EAP influence treatment outcome in multiple myeloma patients undergoing autologous bone marrow transplantation. Int J Cancer 120: 1036-1045, 2007.

17. Vangsted AJ, Klausen TW, Gimsing P, Andersen NF, Abildgaard N, Gregersen H and Vogel U: A polymorphism in NFKB1 is associated with improved effect of interferon-\{alpha\} maintenance treatment of patients with multiple myeloma after high-dose treatment with stem cell support. Haematologica 94: 1274-1281, 2009

18. Badros A, Goloubeva O, Dalal JS, et al: Neurotoxicity of bortezomib therapy in multiple myeloma: a single-center experience and review of the literature. Cancer 110: 1042-1049, 2007. 